\title{
Finite Differences of the Partition Function
}

\section{By Hansraj Gupta}

\begin{abstract}
From the Hardy-Ramanujan-Rademacher formula for $p(n)$-the number of unrestricted partitions of $n$, it is not difficult to deduce that there exists a least positive integer $n_{0}(r)$ such that $V^{r} p(n) \geqslant 0$ for each $n \geqslant n_{0}(r)$, where $V p(n)=$ $p(n)-p(n-1)$ and $V^{r} p(n)=V\left\{V^{r-1} p(n)\right\}$. In this note, we give values of $n_{0}(r)$ for each $r \leqslant 10$ and conjecture that $n_{0}(r) / r^{3} \sim 1$.
\end{abstract}

1. Notation. In the following, small letters denote positive integers unless stated otherwise; $p(n)$ denotes the number of unrestricted partitions of $n$;

$p(n, m)$ is the number of partitions of $n$ into exactly $m$ summands, when $m \leqslant$ $n$; and we take as usual

$$
\begin{gathered}
p(0)=1, \quad p(-n)=0 ; \\
p(n, m)=0 \quad \text { for } n<m ; \quad p(0, m)=0=p(-n, m) .
\end{gathered}
$$

For any arithmetic function $f(n)$, the operator $V$ is defined by

$$
V f(n)=f(n)-f(n-1) \text { and } V^{r} f(n)=V\left\{V^{r-1} f(n)\right\} .
$$

2. Differences of $p(n)$. We have [1]

$$
p(n)-p(n-1)=\sum_{m \geqslant 1} p(n-m, m) \text { for each } n \geqslant 1 ;
$$

so that $V p(n) \geqslant 0$ for $n \geqslant 1$. For $n=0, V p(n)=1$. Again,

$$
V^{2} p(n)=p(n)-2 p(n-1)+p(n-2)
$$

$$
=\sum_{m \geqslant 1}\{p(n-m, m)-p(n-1-m, m)\}, \quad n \geqslant 2 .
$$

Hence, we have the known result

$$
V^{2} p(n) \geqslant 0 \text { for } n \geqslant 2 .
$$

For $n=1$, however, $V^{2} p(n)=-1$. For $n=0, V^{2} p(n)=1$.

Using the well-known Hardy-Ramanujan-Rademacher series for $p(n)$, it is not difficult to show that

$$
V^{r} p(n)=C_{r} p(n)\left(1+O\left(n^{-1 / 2}\right)\right)
$$

Received November 28, 1977.

AMS (MOS) subject classifications (1970). Primary 10A45, $12 \mathrm{H10.}$

Copyright $\odot$ 1978, American Mathematical Society 
where $C_{r}=(\pi / \sqrt{6})^{r} / 4 \sqrt{3}$. Hence, there exists a least positive integer $n_{0}(r)$ such that

$$
V^{r} p(n) \geqslant 0 \text { for each } n \geqslant n_{0}(r) .
$$

More explicitly, on the basis of our calculations, we can say that for each odd $n<n_{0}(r), V^{r} p(n)$ is negative; for each odd $n \geqslant n_{0}(r), V^{r} p(n)$ is $\geqslant 0$; while for each even $n \geqslant 0, V^{r} p(n) \geqslant 0$.

\begin{tabular}{|c|c|c|c|c|c|c|c|}
\hline$r$ & $n_{0}$ & $n$ & $V^{r} p(n)$ & $r$ & $n_{0}$ & $n$ & $V^{r} p(n)$ \\
\hline \multirow[t]{7}{*}{3} & 26 & 21 & -4 & 7 & 352 & 349 & -78036820 \\
\hline & & 23 & -2 & & & 351 & -42437469 \\
\hline & & 25 & -4 & & & 352 & 1774868363 \\
\hline & & 26 & 32 & & & 353 & 865716 \\
\hline & & 27 & 1 & & & 355 & 52281173 \\
\hline & & 28 & 38 & & & 359 & 184178679 \\
\hline & & 29 & 5 & & & & \\
\hline \multirow[t]{5}{*}{4} & 68 & 65 & -87 & 8 & 510 & 509 & -5733970174 \\
\hline & & 67 & -64 & & & 510 & 334894629181 \\
\hline & & 68 & 1497 & & & 511 & 1250502420 \\
\hline & & 69 & 17 & & & 513 & 9319602052 \\
\hline & & 71 & 152 & & & & \\
\hline \multirow[t]{4}{*}{5} & 134 & 129 & -8840 & 9 & 704 & 703 & -457227929371 \\
\hline & & 133 & -3143 & & & 704 & 78392767253289 \\
\hline & & 134 & 112115 & & & 705 & 999762846394 \\
\hline & & 135 & 951 & & & 709 & 4513761223991 \\
\hline \multirow[t]{5}{*}{6} & 228 & 223 & -789593 & 10 & 934 & 933 & -145185040420380 \\
\hline & & 225 & -559660 & & & 934 & 20814672816639740 \\
\hline & & 227 & -247781 & & & 935 & 191102837857344 \\
\hline & & 228 & 12379258 & & & 937 & 566418708656258 \\
\hline & & 229 & 125723 & & & & \\
\hline
\end{tabular}


3. The Table. In the table above, we give the values of $n_{0}(r)$ for $3 \leqslant r \leqslant$ 10. We give also values of $V^{r} p(n)$ for some values of $n$ in the neighborhood of $n_{0}(r)$ to bring out clearly how the change takes place. The Royal Society Tables of Partitions [2] were freely used in preparing this table.

It is noteworthy that within the limits of our table

$$
n_{0}(r) / r^{3} \text { is about } 1 \text {. }
$$

We conjecture that

$$
n_{0}(r) / r^{3} \sim 1
$$

We might here mention that the problem discussed in this note was raised by George E. Andrews.

\section{Panjab University}

Chandigarh 160014, India

402 Mumfordganj

Allahabad 211002 , India 48 \#5995.

1. H. GUPTA, "Two theorems in partitions," Indian J. Math., v. 14, 1972, pp. 7-8. MR

2. H. GUPTA, C. E. GWYTHER \& J. C. P. MILLER, Tables of Partitions, University Press, Cambridge, 1958. 